\title{
Analysis of Time Domain Characteristic of Ultra-high Frequency Antenna Sensor Detecting Partial Discharge
}

\author{
Xuan Feng ${ }^{1, a}$, Yushun Liü, b \\ ${ }^{1}$ Hubei Electric Power Dispatch\&Control Center, No.341 Xudong Street Wuhan, Hubei, China \\ ${ }^{2}$ School of Electrical Engineering, Wuhan University, Wuhan, Hubei, China \\ a314649743@qq.com, bsilencelys@163.com
}

\begin{abstract}
Keywords: Partial discharge; Electromagnetic wave signal; UHF antenna sensor; Time-domain characteristic; Pulse response

Abstract: The analysis of time domain signal is a significance method of pattern recognition and fault degree identification in partial discharge (PD) detection. Frequency domain parameters are usually used to judge the performance of ultra-high frequency (UHF) detecting antenna. However, this method is unable to judge the pulse response characteristic of UHF antenna in time domain. The time domain characteristic test system of PD UHF detecting antenna based on reciprocity theorem for antenna is built in this paper. Three time domain judgment parameters of UHF detecting antenna are proposed. The time domain characteristics of four typical ultra-wide band UHF antennas have been tested. The results show that the ridged horn antenna and the Vivaldi antenna present the excellent time domain characteristics and retain the characteristics of signal source maximally. The pulse response test results of four UHF detecting antennas are verified in simulation PD test.
\end{abstract}

\section{Introduction}

Partial discharge (PD) is the representation of insulation defect in high voltage electrical equipment. The insulation may be breakdown with the development of partial discharge, which leads to high voltage equipment faults. Through detecting and processing the ultra-high frequency (UHF) electromagnetic wave signal radiated from PD source, the type and location of insulation defects can be obtained. This method is known as the UHF method in PD detection. Due to the high sensitivity and strong anti-interference capability, the UHF method has been widely employed in PD detection [1]. In field PD detection, the UHF method can effectively avoid the low-frequency electromagnetic interference and the corona discharge disturbance [2].

Because the frequency range of $\mathrm{UHF}$ is from $300 \mathrm{MHz}$ to $3 \mathrm{GHz}$, the ultra-wide band antenna sensor is regard as the core technology in UHF PD signal detection. The frequency domain parameters are widely used to evaluate antenna sensor performance, such as return loss coefficient $\left(\mathrm{S}_{11}\right)$, voltage standing wave ratio (VSWR), antenna gain, virtual height and so on. These performance evaluation parameters are the functions with frequency variation. However, the time domain performance of antenna sensor determines the response results between antenna and PD pulse, which have great influence on the time domain features of detected UHF PD signal. Based on the Gigahertz transverse electromagnetic (GTEM) cell, M.D. Judd has proposed the time domain characteristic measuring system and corresponding evaluation parameters of UHF PD detection antenna sensor [3]. However, due to the high cost and the large floor space, this method is not applied widely in practice.

Based on the reciprocity theorem for antenna, this paper proposed a novel time domain characteristic measuring system of UHF PD detection antenna sensor. Through analyzing the oscillation time, correlation coefficient and head wave energy ratio of UHF signal, the time domain characteristic of UHF PD detection antenna sensor is evaluated. Four typical UHF PD detection antenna sensors were test in the measuring system to analyze their time domain characteristic. In addition, the comparison results of simulation PD test also proposed the theoretical basis of selecting UHF PD detection antenna sensor. 


\section{Evaluation parameters of time domain characteristic}

Due to the impedance matching discontinuity of UHF detection antenna, electromagnetic wave will be catadioptric inside the antenna sensor. It means that the detected UHF PD signal will be oscillating, which is known as smearing effect. In order to reduce the error of the subsequent signal processing, it is essential to obtain the original PD pulse when using UHF antenna sensor to detect PD signal. However, the existing evaluation parameters in frequency domain can't describe the time domain feature of UHF antenna sensor clearly and completely. Therefore, in order to analyze the time domain characteristic of UHF antenna sensor, it is essential to construct the time domain characteristic measuring system and propose corresponding evaluation parameters. Typical pulse response signal of UHF antenna sensor is given in Figure 1. In this paper, three parameters are proposed to evaluate the time domain characteristic of UHF antenna sensor.

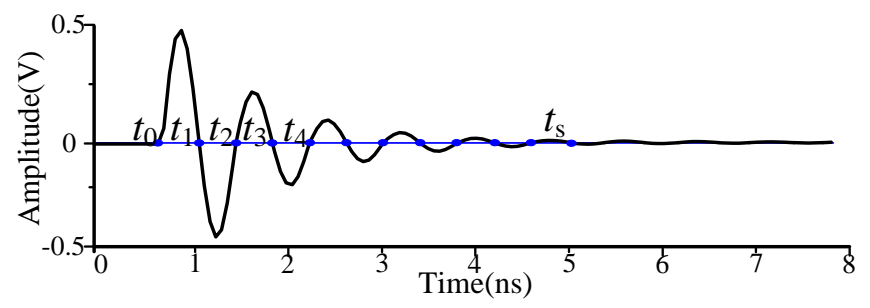

Fig. 1 Impulse response signal of normal ultra-wideband UHF antenna

(1) Oscillation time

Oscillation time $\tau_{p}$ is defined as Equation (1).

$$
\tau_{p}=t_{s}-t_{0}
$$

Oscillation of waveform leads to energy dispersion of detected PD pulse signal and amplitude reduction. This kind of signal waveform distortion aggravates the difficulty of recognizing PD signals. Longer oscillation time leads to worse capacity of recovering original UHF PD signal time domain characteristic using antenna sensor.

(2) Correlation coefficient

Correlation coefficient is an index reflecting the correlation level between different variables. Based on the deviations between two variables and their mean, the correlation level between different variables can be reflected by multiplying the deviations of two variables.

Through calculating the correlation coefficient between the pulse source signal $g(t)$ and pulse response signal $f(t)$, the pulse response characteristic of antenna sensor in time domain can be evaluated. Due to the rapid attenuation of UHF electromagnetic wave signal, the two kinds of signal functions should be normalized for the following calculation of correlation coefficient $r$. The calculation formula of correlation coefficient $r$ is given in Equation (2). The time window length of two signal functions is set to the same, and the initial point is set to the initial point of head wave $t_{0} . x_{i}$ and $y_{i}$ in Equation (2) is the sampling point $i$ numerical value of $g(t)$ and $f(t)$, respectively. $n$ is the number of sampling points.

$$
r=\frac{n \sum_{i=1}^{n} x_{i} y_{i}-\sum_{i=1}^{n} x_{i} \cdot \sum_{i=1}^{n} y_{i}}{\sqrt{n \sum_{i=1}^{n} x_{i}^{2}-\left(\sum_{i=1}^{n} x_{i}\right)^{2}} \cdot \sqrt{n \sum_{i=1}^{n} y_{i}^{2}-\left(\sum_{i=1}^{n} y_{i}\right)^{2}}}
$$

The correlation coefficient between original signal and detected signal directly reflects the recovery ability of PD UHF signal features by using UHF detected antenna. Higher correlation coefficient between the original signal and detected signal detected by UHF antenna sensor reflects the better time domain characteristic of UHF detected antenna and the higher recovery degree of original PD UHF signal. When the original signal and detected signal is the same, the correlation coefficient equals to 1. Meanwhile, the maximum features of original signal are retained, and the analysis results of PD signal will be more accuracy.

(3) Head wave energy ratio 
Due to the multi-path effect of electromagnetic wave and the impedance mismatch in the working bandwidth of UHF antenna, the end of detected impulse response signal will be oscillation. Because most features of original impulse signal exist in the head wave of detected signal, the features of head wave are used to recognize and locate the PD UHF signal usually. Therefore, the ratio between the head wave energy and the energy of whole impulse response signal function $f(t)$ can be used to evaluate the time domain characteristic of UHF antenna sensor. The lower value of head wave energy ratio reflects less feature quantity in original impulse signal. It means that the recovery ability of PD UHF signal will be decreased, and accuracy of PD UHF signal analysis results. The calculation method of head wave energy ratio is given in Equation (3).

$$
E_{\eta}=\frac{\int_{t_{0}}^{t_{2}}|f(t)| \mathrm{d} t}{\int_{t_{0}}^{t_{s}}|f(t)| \mathrm{d} t}
$$

\section{Test system of time domain characteristic}

3.1 Test system. Based on the antenna reciprocity theorem, the characteristic parameters of receiving antenna is the same as the parameters of transmitting antenna, such as working bandwidth, radiation pattern, antenna gain, polarization characteristic and so on. Based on the analytical method of time domain characteristic of antenna in [4], the time domain characteristic test system of UHF antenna is established. The connection diagram of test system is given in Figure 2. Due to the rapid breakdown characteristics of PD, the duration time of PD signal is about a few nanoseconds, and the pulse rise time is about one nanosecond. Therefore, an UHF pulse source which can output Gaussian pulse function was selected for simulating the PD UHF signal in test system. The outputting Gaussian pulse function $g(t)$ is given in Equation (4). The duration time of simulation signal is about $2 \mathrm{~ns}$, and the pulse rise time is $0.6 \mathrm{~ns}$. The outputting amplitude of simulation signal is $-1.52 \mathrm{~V}$, the outputting waveform is given in Figure 3. In the time domain characteristic test system, the pulse source is used to output UHF impulse signal, two same antenna sensors are used to transmit and receive electromagnetic wave. The signal detected by received antenna and oscilloscope is analyzed to evaluate the time domain characteristic of UHF antenna sensor.

$$
g(t)= \pm \frac{1}{\sqrt{2 \pi \sigma^{2}}} \mathrm{e}^{-\frac{t^{2}}{2 \sigma^{2}}}
$$

The high speed digital oscilloscope (LeCory 640Zi) which has 40Gs/s sampling rate and $4 \mathrm{GHz}$ bandwidth was used to obtain the UHF signal detected by UHF antenna sensor. The distance between transmitted and received antennas is $2 \mathrm{~m}$. Because the surrounding metal objects may influence the propagation of electromagnetic wave, the surrounding of two antennas should be ensured no metal object exist. The anechoic chamber is the best environment for this test system.

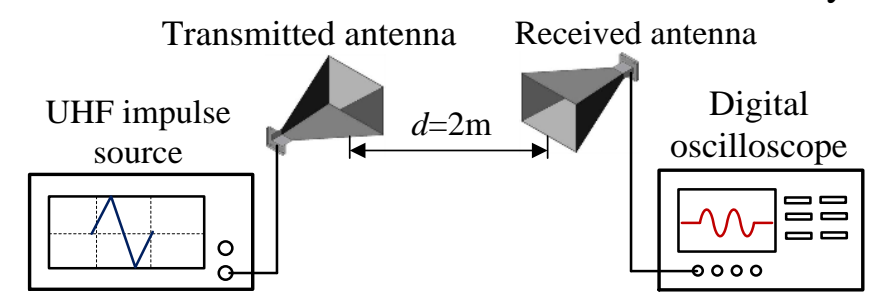

Fig. 2 UHF antenna time-domain characteristics testing system

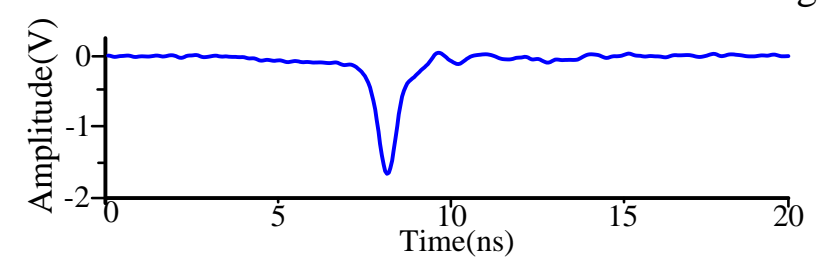

Fig. 3 Gaussian pulse function generated by impulse source 
2.2 Test antennas. Four types of typical UHF antenna were selected to test their time domain characteristic, namely, ridged horn antenna, Vivaldi antenna, micro-strip antenna and helical antenna. The network analyzer (Agilent E8356A) was used to test the return loss $\mathrm{S}_{11}$ of each UHF antenna, the test results are given in Figure 4.

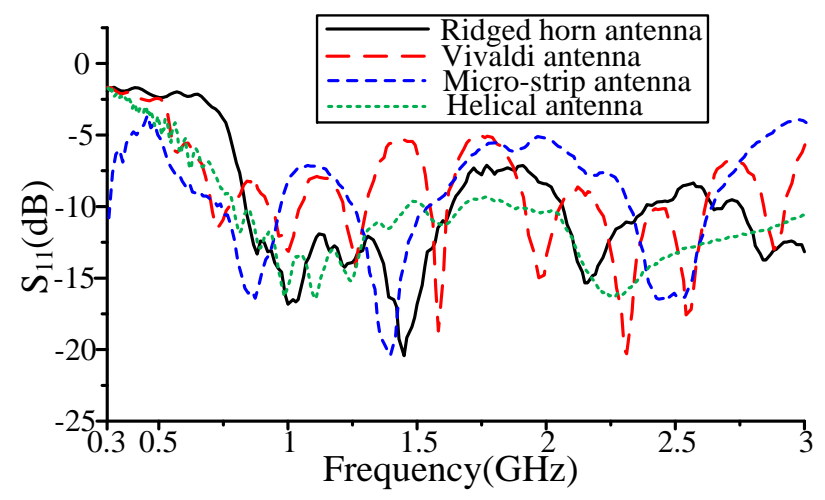

Fig. $4 S_{11}$ parameters of each UHF antenna

2.3 Test results. The test results of four types of typical UHF antenna are given in Figure 5. Based on the test results, time domain characteristic parameters of four types of typical UHF antenna were calculated as shown in Table 1. The results show that the time domain characteristic of Vivaldi antenna is the best and the time domain characteristic of helical antenna is the worst comparatively. The duration time of signal detected by Vivaldi antenna is short, and the head wave can reflect the negative polarity of impulse source. It means that the more accurate original time domain feature of PD signal can be obtain when using Vivaldi antenna to detect PD UHF signal.

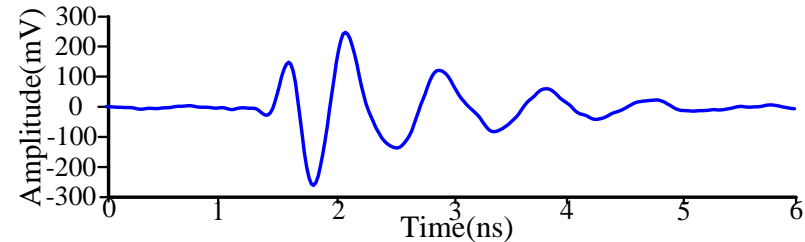

(a) Ridged horn antenna

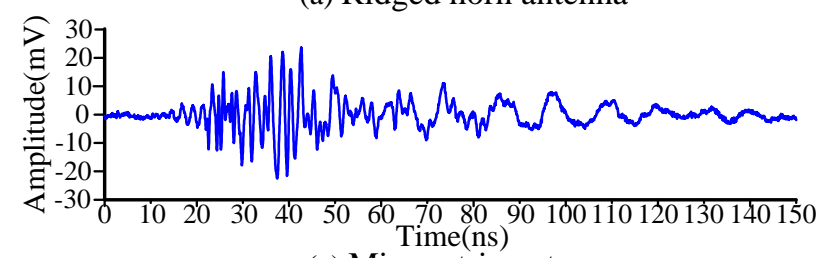

(c) Micro-strip antenna

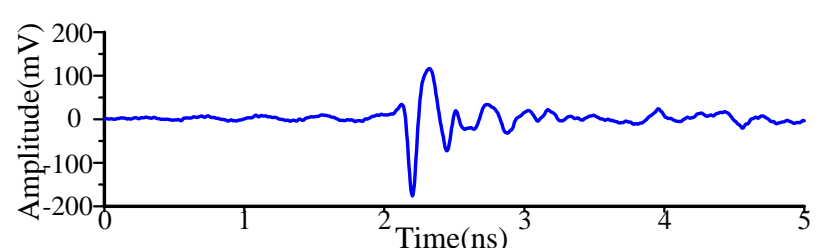

(b) Vivaldi antenna

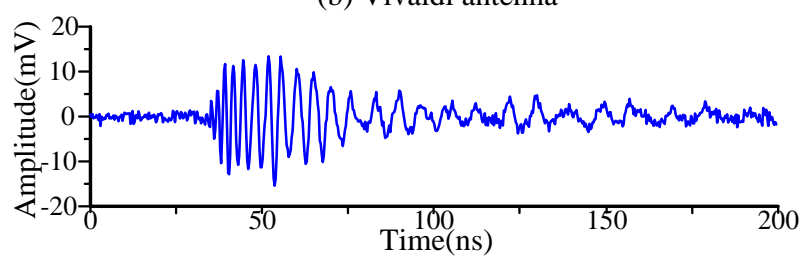

(d) Helical antenna

Fig. 5 Impulse response signal of each UHF antenna

\section{Simulation test of partial discharge}

In order to test the time domain characteristic of four types of typical UHF antenna in practical PD signal detection, the PD test platform based on simulation insulation defect model is established in this paper. Moreover, the PD test results using different UHF antenna were compared and analyzed.

Table 1 Estimate parameters

\begin{tabular}{cccc}
\hline Antenna Type & $\begin{array}{c}\text { Duration } \\
\text { time }\end{array}$ & $\begin{array}{c}\text { Correlation } \\
\text { coefficient }\end{array}$ & $\begin{array}{c}\text { Head wave energy } \\
\text { ratio }\end{array}$ \\
\hline Ridged horn antenna & $4.45 \mathrm{~ns}$ & 0.497 & $40.2 \%$ \\
Vivaldi antenna & $1.9 \mathrm{~ns}$ & 0.599 & $82.8 \%$ \\
Micro-strip antenna & $122 \mathrm{~ns}$ & 0.114 & $8.1 \%$ \\
Helical antenna & $168 \mathrm{~ns}$ & 0.127 & $7.6 \%$ \\
\hline
\end{tabular}


3.1 Test platform. The connection diagram of PD test platform is given in Figure 6 . The test platform was consist of high voltage test power source, simulation insulation defect model, high speed digital oscilloscope (LeCory 640Zi) and above-mentioned four types of UHF antenna sensors. The four antenna sensors were placed in parallel, and the distance between antenna sensors and insulation defects are all $2 \mathrm{~m}$. Four UHF signals were transmitted to the oscilloscope using the same coaxial cables.

In order to obtain the PD UHF signal which close to the reality. The air gap partial discharge model was selected as the simulation insulation defect model in this paper. This insulation defect model was widely used in simulation PD signal test. The structure of insulation defect model is given in Figure 7. The insulation defect model was made by epoxy resin, and the diameter and thickness of interior air gap is $5 \mathrm{~mm}$ and $2 \mathrm{~mm}$, respectively.

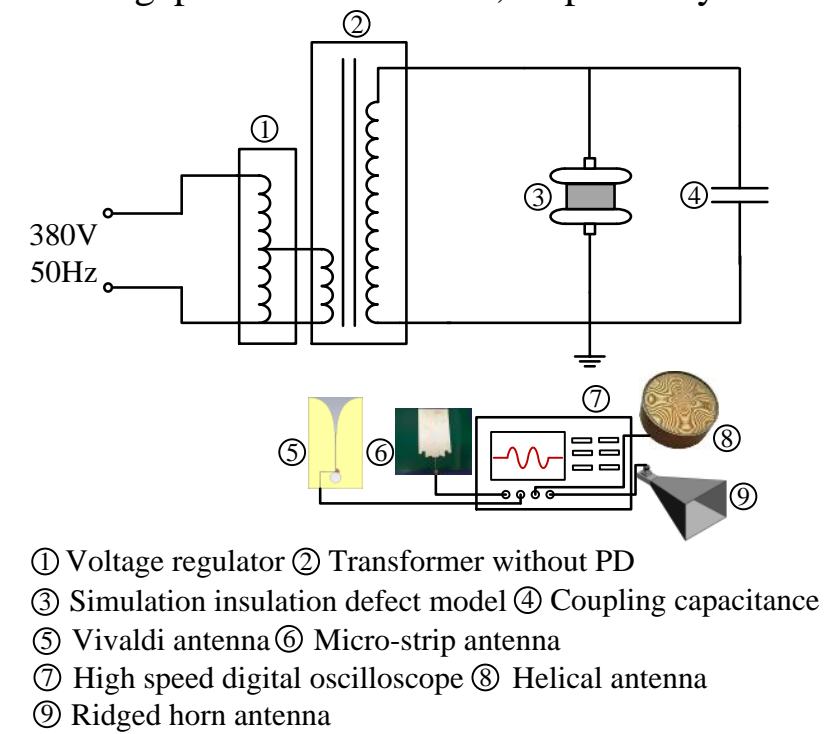

Fig. 6 PD test platform in laboratory

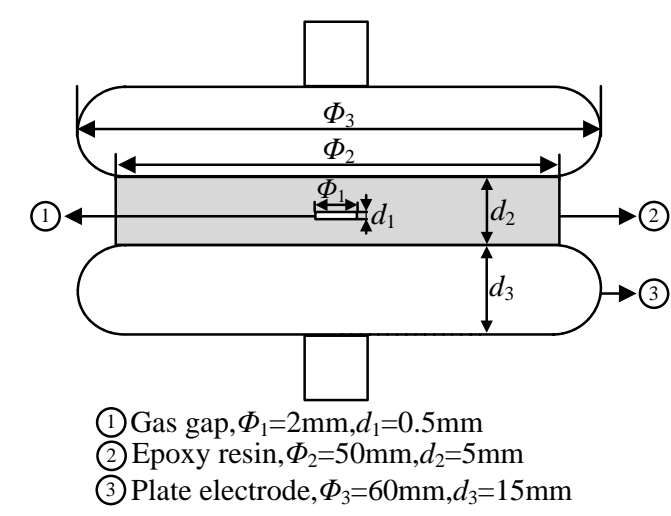

Fig. 7 Air gap defect discharge model

3.2 Test results. When the impressed voltage is $11.2 \mathrm{kV}$, the PD appeared in insulation defect model. The time domain waveforms of PD UHF signals detected by four UHF antenna sensors are given in Figure 8 . The peak value, duration time and head wave energy ratio of four PD signal were calculated and shown in Table 2. The results show that the time domain characteristics of ridged horn antenna and Vivaldi antenna are relatively better. The test results of time domain characteristic and PD detection are corresponding, which means that ridged horn antenna and Vivaldi antenna are more suitable to detect PD UHF signal.

Table 2 Estimate parameters of four PD signal

\begin{tabular}{cccc}
\hline Antenna Type & Peak value & $\begin{array}{c}\text { Duration } \\
\text { time }\end{array}$ & Head wave energy ratio \\
\hline Ridged horn antenna & $26.7 \mathrm{mV}$ & $7.3 \mathrm{~ns}$ & $21.6 \%$ \\
Vivaldi antenna & $21.8 \mathrm{mV}$ & $4.6 \mathrm{~ns}$ & $27.8 \%$ \\
Micro-strip antenna & $8.3 \mathrm{mV}$ & $15.8 \mathrm{~ns}$ & $12.7 \%$ \\
Helical antenna & $7.8 \mathrm{mV}$ & $26.6 \mathrm{~ns}$ & $5.2 \%$ \\
\hline
\end{tabular}




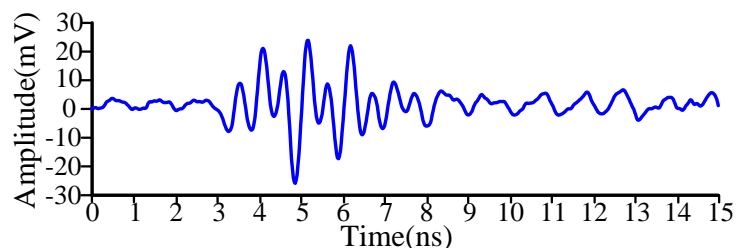

(a) Ridged horn antenna

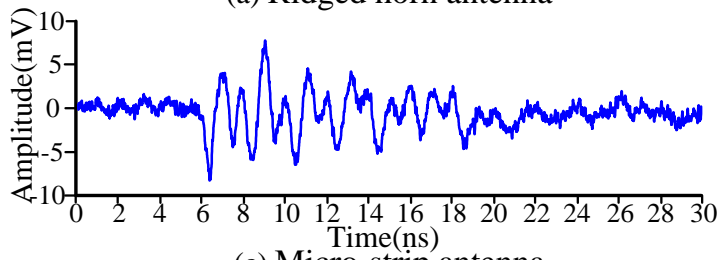

(c) Micro-strip antenna

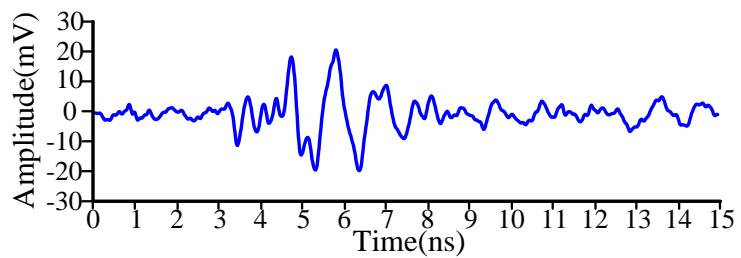

(b) Vivaldi antenna

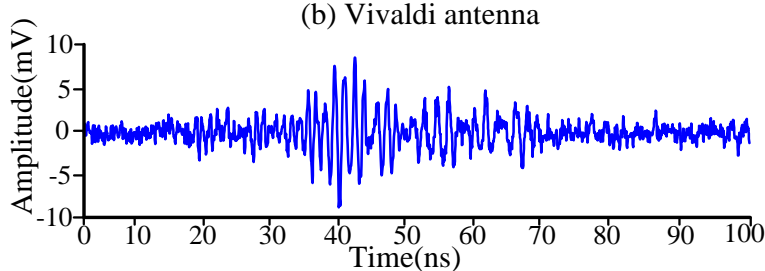

(d) Helical antenna

Fig. 8 PD test platform in laboratory

\section{Conclusions}

(1) The test system of time domain characteristic is established based on the antenna reciprocity theorem, three evaluation parameters, namely, oscillation time, correlation coefficient and head wave energy ratio are proposed to evaluate the time domain characteristic of UHF antenna sensor.

(2) Based on the evaluation method of time domain characteristic, the time domain characteristics of ridged horn antenna, Vivaldi antenna, micro-strip antenna and helical antenna are evaluated. The results show that the ridged horn antenna and Vivaldi antenna have better time domain characteristic, which retain more features of original impulse signal.

(3) The PD test results and time domain characteristic analysis results are corresponding, which proves the effectiveness of proposed time domain characteristic evaluation method. The ridged horn antenna and Vivaldi antenna are more suitable to detect PD UHF signal.

\section{References}

[1] Jiang, T., Li, J., Zheng, Y., et al. Improved bagging algorithm for pattern recognition in UHF signals of partial discharges, 2011, Energies, 4, (7), pp. 1087-1101

[2] Hou, H., Sheng, G., Jiang, X. Robust time delay estimation method for locating UHF signals of partial discharge in substation. IEEE Trans. Power Del., 2013, 28, (3), pp. 1960-1968

[3] Judd M D, Farish O. A pulsed GTEM system for UHF sensor calibration. IEEE Transactions on Instrumentation and Measurement, 1998, 47(4): 875-880.

[4] Caratelli D, Yarovoy A. Unified time-and frequency-domain approach for accurate modeling of electromagnetic radiation processes in ultrawideband antennas. IEEE Transactions on Antennas and Propagation, 2010, 58(10): 3239-3255. 